\title{
Physiologic Cervical Alignment Change between Cervical Spine X-ray and Computed Tomography
}

\author{
Ho Jin Lee, II Sup Kim, ${ }^{1}$ Jae Taek Hong ${ }^{2}$ \\ Department of Neurosurgery, St. Vincent Hospital, College of Medicine, The Catholic University of Korea, Suwon, Korea \\ Department of Neurosurgery, ${ }^{2}$ Eunpyeong St. Mary's Hospital, College of Medicine, The Catholic University of Korea, Seoul, Korea
}

Objective : The purpose of this study was to investigate the correlations among various radiological parameters used to determine cervical alignment from cervical spine radiographs (X-CS) and cervical spine computed tomography (CT-CS), both within and between modalities.

Methods : This study included 168 patients ( $\leq 60$ years old) without a definite whole spine deformity who underwent CT-CS and X-CS. We measured occipital slope (O-s), C1 slope, C2 slope, C7 slope, sella turcica - C7 sagittal vertical axis (StC7-SVA), spinocranial angle, T1 slope, and C27-SVA. We calculated the O-C2 angle, O-C7 angle, and C2-7 angle from the measured parameters and conducted correlation analyses among multiple parameters.

Results : The intrinsic correlation features among multiple cervical parameters were very similar for both X-CS and CT-CS. The two SVA parameters (C27-SVA and StC7-SVA) were mainly influenced by the upper cervical slope parameters $(r=|0.13-0.74|)$ rather than the lower slope cervical parameters $(r=|0.08-0.13|)$. The correlation between $\mathrm{X}-\mathrm{CS}$ and $\mathrm{CT}-\mathrm{CS}$ for each radiological parameter was statistically significant $(r=0.26-0.44)$ except for $0-s(r=0.10)$ and StC7-SVA $(r=0.11)$.

Conclusion : The correlation patterns within X-CS and CT-CS were very similar in this study. The correlation between X-ray and CT was statistically significant for most radiological parameters, and the correlation score increased when the horizontal gaze was consistently maintained. The lower cervical parameters were not statistically associated with translation-related parameters (C2-7 SVA and StC7-SVA). Therefore, the upper cervical segment may be a better predictor for determining head and neck translation.

Key Words : Cervical spine alignment $\cdot X$-ray · Computed tomography.

\section{INTRODUCTION}

Standing cervical spine radiographs (X-CS) are considered the standard protocol to evaluate cervical alignment, and various radiological parameters can be used to determine sagittal balance both quantitatively and qualitatively. Using X-CS, many popular cervical parameters (e.g., C2-7 angle [C2-7A], sagittal vertical axis [SVA], C1-slope [C1-s], C2-slope [C2-s], and C7-slope [C7-s]) are easily measured to analyze cervical balance. However, the cervical spine characteristically serves as a support for the head (horizontal gaze) and is closely related to the thoracic spine anatomically. Therefore, additional

- Received : November 17, 2020 • Revised : December 17, 2020 •Accepted : January 13, 2021

- Address for reprints : II Sup Kim

Department of Neurosurgery, St. Vincent Hospital, College of Medicine, The Catholic University of Korea, 93 Jungbu-daero, Paldal-gu, Suwon 16247, Korea Tel : +82-31-249-7196, Fax : +82-31-249-5208, E-mail : nsman72@hanmail.net, ORCID : https://orcid.org/0000-0001-6328-2955 
parameters that include the head and thoracic spine (e.g., $\mathrm{O}-$ slope, O-C7 angle [O-C7A], T1-slope [T1-s], thoracic inlet angle [TIA], neck tilt [NT]), are necessary and are used frequently. In particular, TIA and T1-s have recently been identified as key factors influencing cervical sagittal alignment ${ }^{11)}$.

It is typically straightforward to measure the parameters associated with the head, but measuring parameters associated with the thoracic vertebrae (i.e., TIA and T1-s) often results in high uncertainty or is impossible to perform. This is due to anatomical interference of the shoulder contour and the ambiguous anatomical marker of the tip of the sternum ${ }^{14)}$. The C7-s is also often difficult to distinguish ${ }^{12)}$. At this time, that the development of a new modality that overcomes the limitations of X-rays and clearly shows the anatomical structures is necessary. Although two commonly used representative modalities (computed tomography [CT] and magnetic resonance image [MRI]) have the power overcome the above limitations, they are typically conducted in the supine position. Therefore, until standing CT or MRI are commercially available, it is necessary to complement the shortcomings of X-ray by using conventional CT or MRI to analyze cervical alignment ${ }^{3}$.

Previous studies have tried to confirm the reliability or validity of the two modalities (CT and MRI); however, the number of patients was relatively small, and some reports were conducted with patients with spinal pathologic lesions ${ }^{12}$. Moreover, the diversity of the analyzed parameters and the degree of precision was relatively low.

The purpose of this study was to investigate the degree of correlation and change of cervical alignment through the $\mathrm{X}$-CS and CT, both within and between the two methods.

\section{MATERIALS AND METHODS}

This study was approved by the Institutional Review Board of The Catholic University of Korea (VC19RESI0211).

\section{Study patients}

This study was conducted using retrospective data analysis of consecutive patients who exhibited minor head or neck injury without definitive neurological symptoms. We evaluated lateral X-CS and cervical spine CT (CT-CS) taken between January 2016 and December 2018.

A total of 168 patients (age range, 20-60 years) who had no other spinal pathology and exhibited relatively well-balanced global spine alignment were included in this study. Patients who had obvious regional or global spinal imbalance (scoliosis angle $>10^{\circ}$ or C7-S1 SVA $>50 \mathrm{~mm}$ ), a major spine injury (fracture or cord injury), a previous spinal operation, or another related medical disease (rheumatoid arthritis or neuromuscular disease) were excluded from the study.

\section{Radiographic evaluation}

The X-CS was collected in the standing position with patients receiving guidance on maintaining a comfortable, straight, horizontal gaze. The CT-CS scans were collected in the supine position, and two representative images (scout lateral image or reconstructed mid-sagittal images) were used for the analysis. The two modalities (X-CS and CT-CS) were typically conducted within a few days; however, patients whose time between the two tests was more than 3 weeks were excluded from this study.

\section{Radiographic analysis}

Various cervical alignment parameters were measured and calculated in this study (Fig. 1). 1) Occipital slope (O-s; hori-

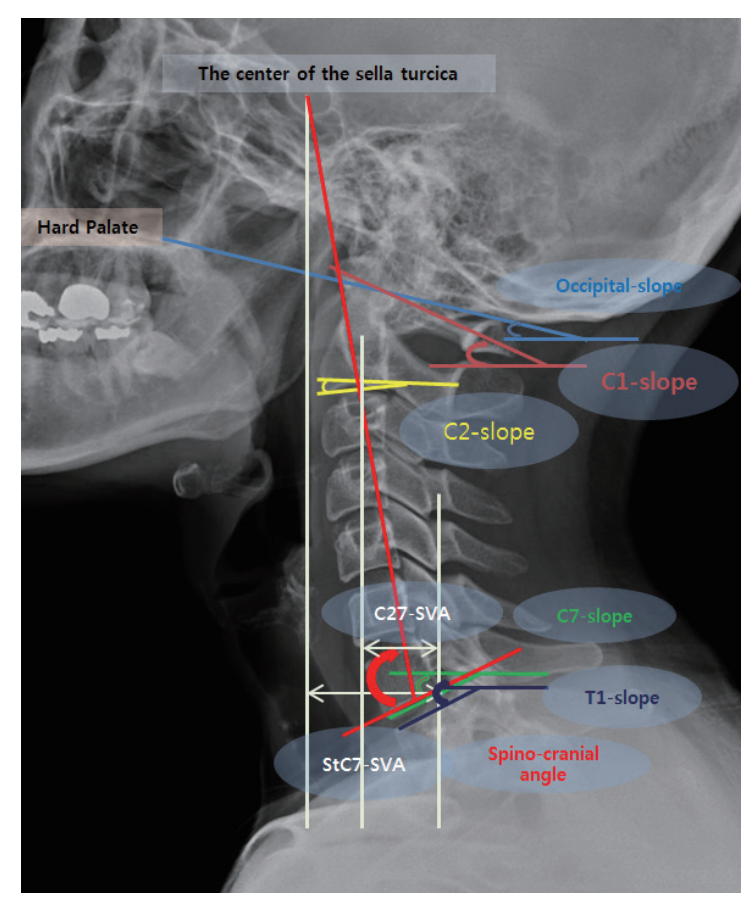

Fig. 1. The measured cervical parameters in this study; occipital slope, C1-slope, C2-slope, C7-slope, T1-slope, C2-C7-sagital vertical axis (C27SVA), sella turcia-C7 sagittal vertical axis (StC7-SVA) and spino-cranial angle. 
Table 1. The change in the cervical spine alignment from the upright (X-CS) to supine position (CT-CS)

\begin{tabular}{|c|c|c|c|c|}
\hline & $\mathrm{X}-\mathrm{CS}$ & CT-CS & Difference between X-CS and CT-CS $(\Delta)$ & $p$-value \\
\hline Occipital slope $\left({ }^{\circ}\right)$ & $11.3 \pm 7.3$ & $-2.2 \pm 5.6$ & -13.5 & $<0.0001$ \\
\hline C1-slope $\left(^{\circ}\right)$ & $19.0 \pm 8.0$ & $4.4 \pm 6.8$ & -14.6 & $<0.0001$ \\
\hline C2-slope $\left({ }^{\circ}\right)$ & $-7.9 \pm 8.0$ & $-12.2 \pm 7.7$ & -4.3 & $<0.0001$ \\
\hline C7-slope $\left(^{\circ}\right)$ & $-22.6 \pm 6.5$ & $-15.2 \pm 7.1$ & 7.4 & $<0.0001$ \\
\hline T1-slope $\left(^{\circ}\right)$ & $-24.6 \pm 6.2$ & $-19.0 \pm 6.5$ & 5.6 & $<0.0001$ \\
\hline C27-SVA (mm) & $16.2 \pm 9.6$ & $15.0 \pm 7.3$ & 1.2 & 0.4902 \\
\hline StC7-SVA (mm) & $26.6 \pm 15.4$ & $40.5 \pm 11.6$ & -13.9 & $<0.0001$ \\
\hline O-C7angle $\left(^{\circ}\right)$ & $34.0 \pm 10.4$ & $13.0 \pm 10.0$ & -21.0 & $<0.0001$ \\
\hline O-C2 angle $\left({ }^{\circ}\right)$ & $19.2 \pm 8.3$ & $9.9 \pm 8.8$ & -9.3 & $<0.0001$ \\
\hline C2-7 angle $\left(^{\circ}\right)$ & $14.7 \pm 11.0$ & $3.0 \pm 10.9$ & -11.7 & $<0.0001$ \\
\hline Spino-cranial angle $\left(^{\circ}\right)$ & $105.3 \pm 8.0$ & $93.0 \pm 8.0$ & -12.3 & $<0.0001$ \\
\hline
\end{tabular}

Values are presented as mean \pm standard deviation. X-CS : cervical spine radiograph, CT-CS : cervical spine computed tomography, SVA : sagittal vertical axis, St : sella turcica

zontal gaze value) : Angle between the McGregor line and a horizontal reference line. 2) C1-s : Angle between the $\mathrm{Cl}$ line (between the middle of the anterior arch and the middle of the posterior arch) and a horizontal reference line. 3) C2-s : Angle between the lower endplate of the C2 body and a horizontal reference line. 4) C7-s : Angle between the lower endplate of the C7 body and a horizontal reference line. 5) T1-s : Angle between the upper endplate of the $\mathrm{T} 1$ body and a horizontal reference line. 6) $\mathrm{O}-\mathrm{C} 2$ angle $(\mathrm{O}-\mathrm{C} 2 \mathrm{~A})$ : Calculated as the O-slope minus the C2-s. 7) O-C7A (global cervical lordosis) : Calculated as the O-slope minus the C7-s. 8) C2-7A (cervical lordosis) : Calculated as the C2-s minus the C7-s. 9) C2C7 SVA (C27-SVA; neck translation) : Distance between a plumb line dropped from the centroid of $\mathrm{C} 2$ and the posterior superior corner of C7. 10) Sella turcica (St) - C7 SVA (StC7SVA; head translation): Distance between a plumb line dropped from the center of the St and the posterior superior corner of C7. And 11) Spino-cranial angle (SCA) : Angle between the $\mathrm{C} 7$ line and the line joining the center of the St and $\mathrm{C} 7$ reference point (the center of the lower endplate of the $\mathrm{C} 7$ body $)^{8)}$.

The slope angle was positive when the specific line was oriented upward from the horizontal line, and lordotic cervical angle was also defined as a positive value. Correlation analyses among multiple variables were performed for X-ray and CT, both within and between groups. All parameters were measured using m-view software version 5.4 (Marosis Technologies, Inc., Seoul, Korea). To reduce measurement error, three observers determined the anatomical landmarks (e.g., the hard palate, the center of the St, C2 centroid, posterosuperior margin of $\mathrm{C} 7$ vertebral body) by mutual consent. To reduce measurement error and increase reliability, all scans were doubled in size.

\section{Statistical analysis}

Data were analyzed using SAS software, version 9.3 (SAS Institute INC., Cary, NC, USA). The Wilcoxon rank-sum test (i.e., Mann-Whitney U test), the paired t-test, and the Pearson correlation coefficient were used for this study. Differences were considered significant at $p$-values $<0.05$.

\section{RESULTS}

The mean age was 38.4 years old (standard deviation, 12.6), and there were 127 men and 41 women. The change in the cervical spine from the upright (X-CS) to supine position (CT$\mathrm{CS})$ induced downward movement $\left(\Delta \mathrm{O}-\mathrm{s}, 13.5^{\circ}\right.$; $\Delta \mathrm{Cl}-\mathrm{s}, 14.6^{\circ}$; $\Delta \mathrm{C} 2$-s, $\left.4.3^{\circ}\right)$ in the higher cervical region and upward movement $\left(\Delta \mathrm{C} 7-\mathrm{s}, 7.4^{\circ} ; \Delta \mathrm{T} 1-\mathrm{s}, 5.6^{\circ}\right)$ in the lower cervical region. As a result, a significant kyphotic change of whole cervical curvature $\left(\Delta \mathrm{O}-\mathrm{C} 7 \mathrm{~A}, 21.0 ; \Delta \mathrm{O}-\mathrm{C} 2 \mathrm{~A}, 9.3 ; \Delta \mathrm{C} 2-7 \mathrm{~A}, 11.7^{\circ}\right)$ and forward head translation (StC7-SVA, $13.9 \mathrm{~mm}$ ) occurred. However, neck translation (C27-SVA) values were maintained without significant change $\left(\Delta 0.3^{\circ}\right)$ (Table 1$)$.

The results of the correlation analysis on the relations 
Table 2. The intrinsic correlation features among multiple cervical parameters in both X-ray and CT

\begin{tabular}{|c|c|c|c|c|c|c|c|c|c|c|c|}
\hline X-ray/CT & O-slope & C1-slope & C2-slope & C7-slope & T1-slope & C27-SVA & StC7-SVA & O-C7 angle & O-C2 angle & C2-7 angle & SCA \\
\hline O-slope & $1 / 1$ & $0.70^{*} / 0.51^{*}$ & $0.42^{*} / 0.16^{*}$ & $-0.13 /-0.22^{*}$ & $-0.12 /-0.28^{*}$ & $-0.25 * /-0.13$ & $-0.73^{*} /-0.52^{*}$ & $0.78 * / 0.72^{*}$ & $0.47^{*} / 0.49^{*}$ & $0.38 * / 0.26^{*}$ & $0.54^{*} / 0.49^{*}$ \\
\hline C1-slope & & $1 / 1$ & $0.68^{*} / 0.39^{*}$ & $-0.12 /-0.11$ & $-0.15 /-0.20^{*}$ & $-0.37 *-0.38 *$ & $-0.74^{*} /-0.65^{*}$ & $0.57^{*} / 0.37^{*}$ & $-0.04 /-0.01$ & $0.57^{*} / 0.35^{*}$ & $0.55^{*} / 0.41^{*}$ \\
\hline C2-slope & & & $1 / 1$ & $-0.13 /-0.07$ & $-0.17^{*} /-0.02$ & $-0.59^{*} /-0.52^{*}$ & $-0.72 * /-0.52^{*}$ & $0.38 * / 0.14$ & $-0.59 * /-0.77^{*}$ & $0.81 * / 0.76^{*}$ & $0.54^{*} / 0.30^{*}$ \\
\hline C7-slope & & & & $1 / 1$ & $0.81 * / 0.76^{*}$ & $-0.10 /-0.13$ & $-0.01 / 0.05$ & $-0.71 * /-0.83^{*}$ & $0.01 /-0.07$ & $-0.68^{*} /-0.70^{*}$ & $-0.73 * /-0.80^{*}$ \\
\hline T1-slope & & & & & $1 / 1$ & $-0.02 /-0.05$ & $0.08 / 0.11$ & $-0.59 *-0.69 *$ & $0.05 /-0.14$ & $-0.60^{*} /-0.51^{*}$ & $-0.65^{*} /-0.68^{*}$ \\
\hline C27-SVA & & & & & & $1 / 1$ & $0.68^{*} / 0.78^{*}$ & $-0.11 / 0.02$ & $0.34^{*} / 0.37^{*}$ & $-0.36^{*} /-0.28^{*}$ & $-0.32 * /-0.28^{*}$ \\
\hline StC7-SVA & & & & & & & $1 / 1$ & $-0.51 * /-0.33^{*}$ & $0.06 / 0.12$ & $-0.53 *-0.40^{*}$ & $-0.61 * /-0.53 *$ \\
\hline O-C7 angle & & & & & & & & $1 / 1$ & $0.32^{*} / 0.33^{*}$ & $0.70 * / 0.64^{*}$ & $0.84^{*} / 0.85^{*}$ \\
\hline O-C2 angle & & & & & & & & & $1 / 1$ & $-0.44^{*} /-0.50^{*}$ & $-0.05^{*} / 0.04^{*}$ \\
\hline C2-7 angle & & & & & & & & & & $1 / 1$ & $0.83^{*} / 0.74^{*}$ \\
\hline SCA & & & & & & & & & & & $1 / 1$ \\
\hline
\end{tabular}

*Statistical significance. CT : computed tomography, SVA : sagittal vertical axis, SCA : spino-cranial angle

Table 3. The correlation analysis between simple radiographs and CT for each cervical parameter

\begin{tabular}{lccccccccccccc}
\hline & O-slope & C1-slope & C2-slope & C7-slope & T1-slope & C27-SVA & StC7-SVA & $\begin{array}{c}\text { O-C7 } \\
\text { angle }\end{array}$ & $\begin{array}{c}\text { O-C2 } \\
\text { angle }\end{array}$ & $\begin{array}{c}\text { C2-7 } \\
\text { angle }\end{array}$ & $\begin{array}{c}\text { Spino- } \\
\text { cranial angle }\end{array}$ \\
\hline $\begin{array}{l}\text { X-ray vs. CT, whole } \\
\text { patients }\end{array}$ & $r=0.10$ & $r=0.28^{*}$ & $r=0.26^{*}$ & $r=0.43^{*}$ & $r=0.44^{*}$ & $r=0.37^{*}$ & $r=0.11$ & $r=0.33^{*}$ & $r=0.36^{*}$ & $r=0.36^{*}$ & $r=0.38^{*}$ \\
$\begin{array}{l}\text { X-ray vs. CT, } \\
\text { horizontal gaze } \\
\text { fixed }\end{array}$ & $r=0.83^{*}$ & $r=0.76^{*}$ & $r=0.72^{*}$ & $r=0.50^{*}$ & $r=0.49^{*}$ & $r=0.31$ & $r=0.41^{*}$ & $r=0.69^{*}$ & $r=0.70^{*}$ & $r=0.66^{*}$ & $r=0.61^{*}$ \\
\hline
\end{tabular}

*Statistically significance; horizontal gaze fixed : the difference value of occipital slope was less than $5^{\circ}$ between two modalities (X-ray and CT). CT : computed tomography, SVA : sagittal vertical axis, St : sella turcica

among the parameters for X-CS are given in Table 2. The upper cervical parameters (O-s, C1-s, and C2-s) were closely related $(\mathrm{r}=0.42-0.70)$ to each other, and the C7-s was also statistically correlated to T1-s $(r=0.81)$. The two SVA parameters (C27-SVA and StC7-SVA) were closely related to the upper cervical slopes (O-s, C1-s, and C2-s) but not to the lower cervical slopes (C7-s and T1-s). C2-7A was statistically associated with all other measured parameters $(r=|0.34-0.81|)$ in this study. SCA was also statistically associated with all other measured parameters except for OC2-A. Although the correlation values of CT-CS were generally lower than X-CS, the correlation patterns for CT-CS were very similar to those of the X-CS correlation analysis.

The correlation scores between X-CS and CT-CS for each radiological parameter are given in Table 3. All parameters except for O-s and StC7-SVA were significantly correlated between CT and X-ray. The correlation score was higher in the lower cervical segment (C7-s and T1-s; range, 0.43-0.44) than in the upper cervical segment (C1-s and C2-s; range, 0.26$0.28)$.

\section{DISCUSSION}

Recently, many studies have shown that cervical spine alignment is very closely related to pain, neurological function, and health-related quality of life ${ }^{5,8,16,17)}$. Therefore, an appropriate understanding of cervical spine alignment is needed to obtain the best clinical results.

Although conventional CT cannot be a golden standard test for cervical balance analysis, CT can contribute in various ways. First, CT may secure a clearer anatomical landmark than X-ray regardless of posture and body shape. Second, it can be an essential substitute for X-rays in severe traumatic conditions. Third, CT may more accurately reflect lying postures such as sleep and rest. Fourth, it serves as a variant (not 
excessively deformed neck) or dynamic posture change of the cervical spine that can be used to examine the cervical alignment. Finally, supine posture is most often used by spinal surgeons during anterior cervical operations.

Generally, the cervical spine changed to a kyphotic alignment in the CT scans as compared with the X-ray scan. The lordotic angle was reduced by downward tilting of the upper cervical vertebrae and upward titling of the lower cervical vertebrae. Although all the slope parameters had a statistically significant change in angle between the procedures, the relative change of O-s $\left(\Delta 13.5^{\circ}\right)$ and Cl-s $\left(\Delta 14.6^{\circ}\right)$ were the largest, followed by C7-s $\left(\triangle 7.4^{\circ}\right)$ and T-1 $\left(\triangle 5.6^{\circ}\right)$, and the smallest change occurred at C2-s $\left(\triangle 4.3^{\circ}\right)$. The angular changes of T1-s and C7-s were much larger than those seen in many previous studies (CT or MRI, $\Delta 1.1^{\circ}-3.2^{\circ}$ ), although Janusz et al. showed that a flexion posture induced a decrement of T1-s by $6.9^{4,6,9,12,15)}$. These differences are likely because the present study had several advantages in selecting patients : a relatively large number of patients $(n=168)$, younger adult patients $(\leq 60$ years old), no cervical spine pathology, and a well-balanced global spine. Therefore, the present results are also reliable and have strong validity. A majority of the measured parameters showed significant changes between the two modalities (X-ray and CT), and only C27-SVA was not statistically different in this study. Liu et al. ${ }^{12)}$ had similar results when comparing Xray and MRI; however, another study determined that SVA significantly changed between modalities".

The intrinsic correlation features among multiple cervical parameters were very similar in both X-CS and CT-CS. The higher cervical parameters (O-slope, C1-s, and C2-s) were closely related with each other, and the lower cervical parameters (C7 slope and T1 slope) were also closely related with each other. The two SVA parameters (C27-SVA and StC7-SVA) were mainly influenced by the higher cervical slope parameters (O-slope, C1-s, and C2-s) but not by the lower cervical slope parameters (C7-s and T1-s). Moreover, the upper cervical slope parameters were more highly correlated with StC7SVA (head translation, $r=|0.72-0.74|$ ) than with C27-SVA (neck translation, $\mathrm{r}=|0.25-0.59|$ ). Many previous reports have shown a close relationship among T1-s, C2-7A and C27-SVA, however the present study showed different results ${ }^{1,13}$. Weng et al. ${ }^{18)}$ also showed a close relationship between T1-s and C27SVA; however, the correlation value was very low $(\mathrm{r}=0.22)$. Moreover, cranial tilt (based on C2 centroid, $r=0.91$ ) had a very strong correlation with C27-SVA in their study. To solidify present results, we conducted a sub-group analysis comparing the two groups according to T1-s value with a cutoff of $25^{\circ}$. The high-T1-s group (mean, 29.8 $8^{\circ}$ and low-T1-s group (mean, $20.1^{\circ}$ ) has almost the same value of C2-7 SVA (15.2 vs. $15.8 \mathrm{~mm}$ ) in this study.

C2-7A (cervical lordosis) showed statistical correlations with all other cervical parameters ranging between $0.26-0.81$ in this study. Although SCA was introduced as a similar parameter to the SCA of the thoraco-lumbar spine, it is not typically used as a representative parameter for analyzing cervical spine alignment. However, SCA showed a strong relationship with all cervical parameters except for O-C2A in both X-CS and CT-CS in this study. Therefore, SCA is as useful as C2-7A for the analysis of cervical alignment.

Almost all parameters were statistically correlated between X-CS and CT-CS; however O-s (horizontal gaze) and StC7SVA were not. Generally, the correlation values were slightly higher for lower cervical slope parameters (C7-s and T1-s, $\mathrm{r}=0.43-0.44)$ than for the upper cervical slope parameters (C1-s and C2-s, $\mathrm{r}=0.26-0.28)$. The correlation values of the three angular parameters (O-C7A, O-C2A, and C2-7A) were very similar in this study. Because the control of the horizontal gaze may have led to a much different cervical alignment change compared to our previous studies, we conducted a second sub-group analysis of the subjects who maintained a fixed horizontal gaze $\left(n=28, O-s=5^{\circ}\right)$ between two modalities (X-CT and CT-CS) ${ }^{7,10)}$. The correlation values of the fixegazed group $(\mathrm{r}=0.31-0.83)$ were higher than the whole patient population ( $\mathrm{r}=0.11-0.44)$ (Table 3$)$. However, contrary to the whole patient group, C27-SVA was the only parameter not statistically inter-correlated between the two modalities.

It is necessary to expand and interpret the above research results more clinically as follows; 1) by simply measuring the typical parameters of the upper and lower cervical segment (e.g., C2-s and C7-s), the degree of overall cervical alignment can be estimated. 2) It may be very important to conduct the radiographic evaluation without positional error (especially in the upper cervical segment), when measuring SVA parameters that predict the degree of neck pain and quality of life. And 3) although there is a difference in absolute values between the modalities (X-ray and CT), the relationship of parameters within the cervical spine itself is very closed related to each other. 
This study has some limitations. First, we did not measure TIA and NT in this study. This is because TIA has wellknown representative constant parameters, and inter- and intra-subject reliability can be very low in X-CS due to anatomical interference. Second, as this was retrospective study, the control of the neck and head posture may not be accurate and precise across subjects. Third, we did not analyze the status of disc degeneration and facet degeneration, which may influence cervical alignment. Fourth, it should be reconsidered that the patient population in this study was not pure healthy people. Therefore, the explanation for the effect of muscle strain occurring after minor injury is very insufficient. We should be aware that how to and how much to affect present study results by neck muscle sprain of patients after mild injury (selected as the object to study). Usually, it has been a general beliefs or expectations that the lordosis of cervical alignment may be changed after neck injury due to muscle spasm or ligament injury. However, Beltsios et al. ${ }^{2}$ showed that there was no statistical significance regarding the sagittal alignment between patients with $(n=60)$ and without $(n=100)$ cervical spine injury. Therefore, it may be possible to secure some validity for the contents of present study.

\section{CONCLUSION}

Kyphotic changes to the cervical spine in the supine posture were mainly influenced by the upper cervical segment, and the correlation patterns within X-CS and CT-CS were very similar in this study. The inter-correlation between X-ray and CT was reaffirmed for many of the cervical alignment parameters. The lower cervical segment was not statistically associated with translation-related parameters (C2-7 SVA and St-C7 SVA). Therefore, the upper cervical segment may be a better predictor for determining head and neck translation. Additionally, C2-7 angle and SCA showed a strong correlation with almost other cervical parameters in both X-ray and CT.

\section{CONFLICTS OF INTEREST}

No potential conflict of interest relevant to this article was reported.

\section{INFORMED CONSENT}

This type of study does not require informed consent.

\section{AUTHOR CONTRIBUTIONS}

\author{
Conceptualization : HJL, ISK \\ Data curation : HJL, ISK, JTH \\ Formal analysis : HJL \\ Methodology : HJL \\ Project administration : HJL \\ Visualization : HJL \\ Writing - original draft : HJL, ISK \\ Writing - review \& editing : HJL, ISK, JTH
}

\section{ORCID}

$\begin{array}{ll}\text { Ho Jin Lee } & \text { https://orcid.org/0000-0002-9208-5931 } \\ \text { Il Sup Kim } & \text { https://orcid.org/0000-0001-6328-2955 } \\ \text { Jae Taek Hong } & \text { https://orcid.org/0000-0001-6453-0439 }\end{array}$

\section{References}

1. Ames CP, Blondel B, Scheer JK, Schwab FJ, Le Huec JC, Massicotte EM, et al. : Cervical radiographical alignment: comprehensive assessment techniques and potential importance in cervical myelopathy. Spine (Phila Pa 1976) 38(22 Suppl 1) : S149-S160, 2013

2. Beltsios M, Savvidou O, Mitsiokapa EA, Mavrogenis AF, Kaspiris A, Efstathopoulos $N$, et al. : Sagittal alignment of the cervical spine after neck injury. Eur J Orthop Surg Traumatol 23 Suppl 1 : S47-S51, 2013

3. Cheng J, Liu P, Sun D, Ma Z, Liu J, Wang Z, et al. : Correlation of cervical and thoracic inlet sagittal parameters by MRI and radiography in patients with cervical spondylosis. Medicine (Baltimore) 98 : e14393, 2019

4. Janusz P, Tyrakowski M, Glowka P, Offoha R, Siemionow K : Influence of cervical spine position on the radiographic parameters of the thoracic inlet alignment. Eur Spine J 24 : 2880-2884, 2015

5. Jouibari MF, Le Huec JC, Ranjbar Hameghavandi MH, Moghadam N, Farahbakhsh F, Khadivi M, et al. : Comparison of cervical sagittal parameters among patients with neck pain and healthy controls: a comparative cross-sectional study. Eur Spine J 28 : 2319-2324, 2019

6. Jun HS, Chang IB, Song JH, Kim TH, Park MS, Kim SW, et al. : Is it possible to evaluate the parameters of cervical sagittal alignment on cervi- 
cal computed tomographic scans? Spine (Phila Pa 1976) 39 : E630E636, 2014

7. Kim JT, Lee HJ, Choi DY, Shin MH, Hong JT : Sequential alignment change of the cervical spine after anterior cervical discectomy and fusion in the lower cervical spine. Eur Spine J 25 : 2223-2232, 2016

8. Le Huec JC, Saddiki R, Franke J, Rigal J, Aunoble S : Equilibrium of the human body and the gravity line: the basics. Eur Spine J 20 Suppl 5 : 558-563, 2011

9. Lee $\mathrm{HD}$, Jeon $\mathrm{CH}$, Chung NS, Kwon HJ : Comparative analysis of three imaging modalities for evaluation of cervical sagittal alignment parameters: a validity and reliability study. Spine (Phila Pa 1976) 42 : 19011907, 2017

10. Lee HJ, Kim JH, Kim IS, Hong JT : Physiologic cervical alignment change between whole spine radiographs and normal standing cervical radiographs. World Neurosurg 122 : e1222-e1227, 2019

11. Lee SH, Kim KT, Seo EM, Suk KS, Kwack YH, Son ES : The influence of thoracic inlet alignment on the craniocervical sagittal balance in asymptomatic adults. J Spinal Disord Tech 25 : E41-E47, 2012

12. Liu W, Fan J, Bai J, Tang P, Chen J, Luo Y, et al. : Magnetic resonance imaging: a possible alternative to a standing lateral radiograph for evaluating cervical sagittal alignment in patients with cervical disc herniation? Medicine (Baltimore) 96 : e8194, 2017
13. Núñez-Pereira S, Hitzl W, Bullmann V, Meier O, Koller H : Sagittal balance of the cervical spine: an analysis of occipitocervical and spinopelvic interdependence, with C-7 slope as a marker of cervical and spinopelvic alignment. J Neurosurg Spine 23 : 16-23, 2015

14. Park JH, Cho CB, Song JH, Kim SW, Ha Y, Oh JK : T1 slope and cervical sagittal alignment on cervical CT radiographs of asymptomatic persons. J Korean Neurosurg Soc 53 : 356-359, 2013

15. Qiao J, Zhu F, Liu Z, Xu L, Zhu Z, Qian B, et al. : Measurement of thoracic inlet alignment on MRI: reliability and the influence of body position. Clin Spine Surg 30 : E377-E380, 2017

16. Scheer JK, Tang JA, Smith JS, Acosta FL Jr, Protopsaltis TS, Blondel B, et al. : Cervical spine alignment, sagittal deformity, and clinical implications: a review. J Neurosurg Spine 19 : 141-159, 2013

17. Tang JA, Scheer JK, Smith JS, Deviren V, Bess S, Hart RA, et al. : The impact of standing regional cervical sagittal alignment on outcomes in posterior cervical fusion surgery. Neurosurgery 71 : 662-669; discussion 669, 2012

18. Weng C, Wang J, Tuchman A, Wang J, Fu C, Hsieh PC, et al. : Influence of T1 slope on the cervical sagittal balance in degenerative cervical spine: an analysis using kinematic MRI. Spine (Phila Pa 1976) 41 : 185-190, 2016 